\title{
Microarray Analysis of Gene Expression by Ginseng Water Extracts in a Mouse Adrenal Cortex after Immobilization Stress
}

\author{
Young-Ock Kim* and Sang Won Lee \\ Medicinal Crops Division, Ginseng and Medicinal Plants Research Institute Rural Development Administration, Eumseong 369-873, \\ Korea
}

To investigate the effects of repeated immobilization-stress challenge on the the hypothalamic-pituitary-adrenal axis, the genomic transcriptome in the adrenal cortex of immobilization-stressed mouse was analyzed by using a cDNA microarray. Mice were subjected to immobilization stress for $2 \mathrm{~h}$ per day for 5 consecutive d. With a 4.0-fold cutoff of arbitrary criteria, the expression levels of 168 out of 41,174 genes were significantly modulated in the adrenal cortex by stress when comparing the control and experimental groups. These genes were related to apoptosis, cell cycle, immune response, inflammatory responses, and signal transduction, and thus may be used as potential targets for the development of therapeutics for chronic stress or depression. Six significant genes among these were selected for real time polymerase chain reaction analysis to confirm the change of their expression levels. The gene for phospho 1 was also further investigated because its expression showed the greatest fold-change.

Keywords: Panax ginseng, Immobilization, Stress, Hypothalamic-pituitary-adrenal, Oligonucleotide array sequence analysis, Adrenal cortex

\section{INTRODUCTION}

Stress affects our daily lives. Many studies have shown that stress has profound effects on immunological parameters in humans and animals [1]. According to Cannon, homeostasis is the product of multiple physiological systems that maintain a steady-state in an organism [2]. Immobilization (IMO), a restraint stressor, is known to induce both psychological and physical stress which results in a wide range of behavioral and physiological alterations, secretion of stress hormones, and neuronal cell death in the brain [3]. Several research groups have reported on IMO stressrelated changes in specific genes or proteins in organs such as stomach, liver, spleen, hippocampus, and the hypothalamic-pituitary-adrenal axis [4,5]. Corticotrophin releasing hormone is secreted by the hypothalamus and leads to the release of adrenocorticotropic hormone (ACTH) from the pituitary gland. ACTH causes the adrenal gland to synthesize and release catecholamines such as corticosterone, epinephrine, dopamine, and norepinephrine, which are all characteristic stress hormones.

Ginseng has a long history as a traditional medicine in Korea, China, and Japan. Recent studies have shown that ginseng increases resistance to stress, decreases blood pressure, benefits immune functions, and exerts antidepressant effects [6,7]. To date, approximately 70 kinds of saponin have been isolated from Panax ginseng. Most of these are protopanaxdiol and protopanaxtriol, which are aglycones of dammarane-type triterpenoids.

Recently, Cho et al. [8] acquired physicochemical

\footnotetext{
(c) This is an Open Access article distributed under the terms of the Creative Commons Attribution Non-Commercial License (http://creativecommons.org/licenses/by-nc/3.0/) which permits unrestricted non-commercial use, distribution, and reproduction in any medium, provided the original work is properly cited.
}

Received 20 Oct. 2010, Revised 12 Jan. 2011, Accepted 12 Jan. 2011

*Corresponding author

E-mail: kyo9128@korea.kr

Tel: +82-43-871-5585, Fax: +82-43-871-5569 
and spectroscopic data from four major diol-saponins: ginsenosides $\mathrm{Rb} 1, \mathrm{Rb} 2, \mathrm{Rc}$, and $\mathrm{Rd}$. Researche on the anti-stress effects of ginseng suggest that total ginseng saponins are the active components involved in the alleviation of mental and physical stress, and the normalization of work capacity [9]. However, the effects of ginseng on the adrenal cortex have not been studied.

DNA microarray technology, which has recently emerged as a powerful tool, allowed us to simultaneously analyze and compare gene expression in normal and treated cells [10]. We compared gene expression patterns in the adrenal cortex of mice either exposed or not exposed to restraint stress by ginseng treatment. Using oligo microarrays containing 44,000 genes of various functional classes, we found that 119 genes were up-regulated whereas 49 genes were down-regulated. We selected six genes of particular interest and confirmed the changes in the expression patterns of these genes by quantitative real time polymerase chain reaction (RT-PCR).

\section{MATERIALS AND METHODS}

Male CD-1(ICR) mice which were 6 wk old (Jung Ang, Seoul, Korea) were used for all experiments. The animals were randomly divided into four experimental groups and housed for at least $8 \mathrm{~d}$ prior to starting the experiments. They were kept on a 12-h light/dark cycle at a temperature of $23 \pm 1^{\circ} \mathrm{C}$ and given food and water $\mathrm{ad}$ libitum. The body weights of the mice were determined daily, immediately after exposure to $2 \mathrm{~h}$ of repeated IMO, using a digital balance (CAS, Seoul, Korea). The experimental procedures were carried out in accordance with the NIH Guide for Care and Use of Laboratory Animals, and were approved by the Kyung Hee University Institutional Animal Care and Use Committee.

For RT-PCR analysis, $1 \mu \mathrm{g}$ of total RNA was reverse transcribed with $200 \mathrm{U}$ of moloney murine leukemia virus (MMLV) reverse transcriptase (Life Technologies, Carlsbad, CA, USA), in $20 \mu \mathrm{L}$ of reaction mixture containing random hexamers (Bioneer, Daejeon, Korea). One microliter of cDNA mixture was then used as DNA templates in $20 \mu \mathrm{L}$ of reaction mixture containing 10 $\mathrm{mM}$ Tris- $\mathrm{HCl}$ (pH8.3), $50 \mathrm{mM} \mathrm{KCl}, 1.5 \mathrm{mM} \mathrm{MgCl}_{2}, 0.2$ $\mathrm{mM}$ dNTP, $0.4 \mathrm{uM}$ each primer, and $0.5 \mathrm{U}$ Taq polymerase (TaKaRa, Shiga, Japan). RT-PCR was performed using a PTC-100 programmable thermal controller (MJ Research, Waltham, MA, USA). All primers were designed using web-based primer selection software, Primer 3 (The Whitehead Institute for Biomedical Research, Cambridge, MA, USA). The primer sequences and reaction condi- tions of the genes are shown in Table 5. The PCR products were separated on $1 \%$ agarose gels, stained with ethidium bromide, and photographed. The intensities of the bands were measured by ImageMaster VDS (Amersham Pharmacia, Buckinghamshire, UK) with ImageMaster TotalLab image-analyzing software. The signals of target PCR bands were normalized against the band intensity of glyceraldehyde-3-phosphate dehydrogenase. Data was expressed as the mean \pm standard error. Differences were analyzed with Student's $t$-test. A $p$-value of $<0.05$ was considered to be significant.

The ten mice of the IMO group were stressed daily by immobilization for $2 \mathrm{~h}$ in Rodent Restraint Cones (Stoelting, Wood Dale, IL, USA) which prevented all forward/ backward and lateral movements. For repeated IMO stress, mice were immobilized at the same time every morning for five consecutive days. Mice were sacrificed by decapitation immediately after the 5th IMO stress treatment on the final day. The adrenal cortex was quickly removed and stored in cold RNAlater solution (Ambion, Austin, TX, USA) for further experimentation.

\section{Real time polymerase chain reaction}

Reverse transcription was performed using a TaqMan Reverse Transcription Regents Kit (Applied Biosystems, Foster City, CA, USA). The cDNA samples were diluted $1,0.5,0.250 .125$, and $0.0625 \mathrm{ng} / \mathrm{mL}$. Triplet quantitative assays were performed on $1 \mathrm{~mL}$ of each cDNA dilution using the SYBR Green Master mix with an ABI 7900 sequence detection system according to the manufacture's protocol (Applied Biosystems). Gene-specific primers were designed by using PRIMEREX PRESS 1.0 software (Applied Biosystems) and are listed in Table 5.

\section{Microarray analysis}

For control and test RNAs, the synthesis of target cRNA probes and hybridization were performed using a Low RNA Input Linear Amplification kit (Agilent Technology, Santa Clara, CA, USA) according to the manufacturer's instructions. Briefly, $1 \mathrm{mg}$ each of total RNA and T7 promoter primer was mixed and incubated at $65^{\circ} \mathrm{C}$ for 10 min. A cDNA master mix (5x first strand buffer, $0.1 \mathrm{M}$ DTT, $10 \mathrm{mM}$ dNTP mix, RNase-Out, and MMLV-RT) was prepared and added to the reaction mixture. The samples were incubated at $40^{\circ} \mathrm{C}$ for $2 \mathrm{~h}$, and then the RT and dsDNA synthesis was terminated by incubating the samples at $65^{\circ} \mathrm{C}$ for $15 \mathrm{~min}$. The transcription master mix was prepared according to the manufacturer's protocol (4x transcription buffer, 0.1M DTT, NTP mix, $50 \%$ PEG, RNase-Out, inorganic pyrophosphatase, T7- 
RNA polymerase, and cyanine $3 / 5-\mathrm{CTP}$ ). Transcription of dsDNA was performed by adding the transcription master mix to the dsDNA reaction samples and incubating at $40^{\circ} \mathrm{C}$ for $2 \mathrm{~h}$ Amplified and labeled cRNA was purified on cRNA Cleanup Module (Agilent Technology) according to the manufacturer's protocol. Labeled target cRNA was quantified using an ND-1000 spectrophotometer (NanoDrop Technologies Inc., Wilmington, DE, USA). After checking the labeling efficiency, fragmentation of cRNA was performed by adding 10x blocking agent and $25 \mathrm{x}$ fragmentation buffer, and incubating at $60^{\circ} \mathrm{C}$ for $30 \mathrm{~min}$. The fragmented cRNA was resuspended in $2 \mathrm{x}$ hybridization buffer and directly pipetted onto an assembled Whole Rat Genome Oligo Microarray (44K; Agilent Technology). The arrays hybridized at $65^{\circ} \mathrm{C}$ for $17 \mathrm{~h}$ using an Agilent Hybridization oven (Agilent Technology). The hybridized microarrays were washed according to the manufacturer's washing protocol.

\section{Data acquisition and analysis}

The hybridized images were scanned using a DNA microarray scanner and quantified with Feature Extraction Software (Agilent Technology). All data normalization and selection of fold-changed genes were performed using GeneSpringGX 7.3 (Agilent Technology). Intensity-dependent normalization (locally-weighted regression scatterplot smoother) was performed where the ratio was reduced to the residual of the Lowess fit of the intensity versus the ratio curve. The averages of the normalized ratios were calculated by dividing the average of normalized signal channel intensity by the average of normalized control channel intensity. Functional annotation of the genes was performed using GeneSpringGX 7.3 according to the Gene Ontology Consortium. Gene classification was based on searches of BioCarta, GenMAPP, DAVID, and Medline databases.

\section{RESULTS AND DISCUSSION}

\section{Microarray}

Microarray analysis is a technique that has been shown to be useful for the simultaneous profiling of global gene expression and converging new genes or new functions of known genes [11]. For these reason, microarray analysis has been used to study gene expression in stressed mice following treatment with herbal medicine [12] and stress-related changes in gene expression following treatment with ginseng.

\section{RNA quality}

Preparation of highly pure undegraded total RNA is essential for performing a successful microarray. The quality of the total RNA was monitored with a RNA 6000 Pico Assay Solution using 2100 Bioanalyzer (Agilent Technology). No degradation was evident in the gel-like images of the control and sample preparations (Fig. 1A). The rRNA ratios (28S/18S) of each preparation were close to 2.0 (Fig. 1B). These data indicate that high-quality RNA was recovered.

\section{MA-plot}

An MA-plot of the microarray data, where $\mathrm{M}$ and A represent $\log 2(\mathrm{Cy} 5$ intensity/Cy3 intensity) and $1 / 2 \log 2$ (Cy5 intensity $\times \mathrm{Cy} 3$ intensity), respectively, is a plot of log-ratio of two expression intensities versus the mean log-expression of the two [13]. As shown in Fig. 2 , most $\mathrm{M}$ values are between \pm 1.0 , meaning that up- or down-regulation scales are mostly less than 2 -fold.

\section{Data processing}

Array images were analyzed to check general signal intensities from the DNA chips after hybridization of the samples and probes on the chips (data not shown). After acquisition from the images, the signals were transformed into digital numbers and an analysis was performed. To test the reproducibility of each microarray analysis, the absolute data from each chip (data not shown) were compared. Normalization and comparison of the data were done and hierarchical clustering was performed.

\section{Functional distribution and regulation of genes}

Genes were classified into nine functional groups (immune response, neurogenesis, cell cycle, apoptosis, DNA repair, signal transduction, and gene transcription). The overall distribution of the genes in each groups that were up- or down-regulated by more than 2-fold following ginseng extract administration on the 5th day after IMO stress are shown in Fig. 3. There were more up-regulated (119) than down-regulated (49) genes. Lists of genes in each group are shown in Table 4.

\section{Stress response-related genes (Table 1) \\ Defension beta 3 (Def 33 )}

The Def $\beta 3$ gene was up-regulated by $4.2 \pm 0.16$ fold. Mammalian cells express a number of peptide antibiotics as an innate host defense system [14]. Defensins and acathelicidin are the two major classes of antimicrobial peptides in humans [15]. Defensins are divided into $\alpha$-and $\beta$-denfensins. In humans, $\alpha$-denfensins are expressed in the small intestine (neurtophils and Paneth cells) whereas $\beta$-denfensins are mainly found in epithelial tissues. 
A

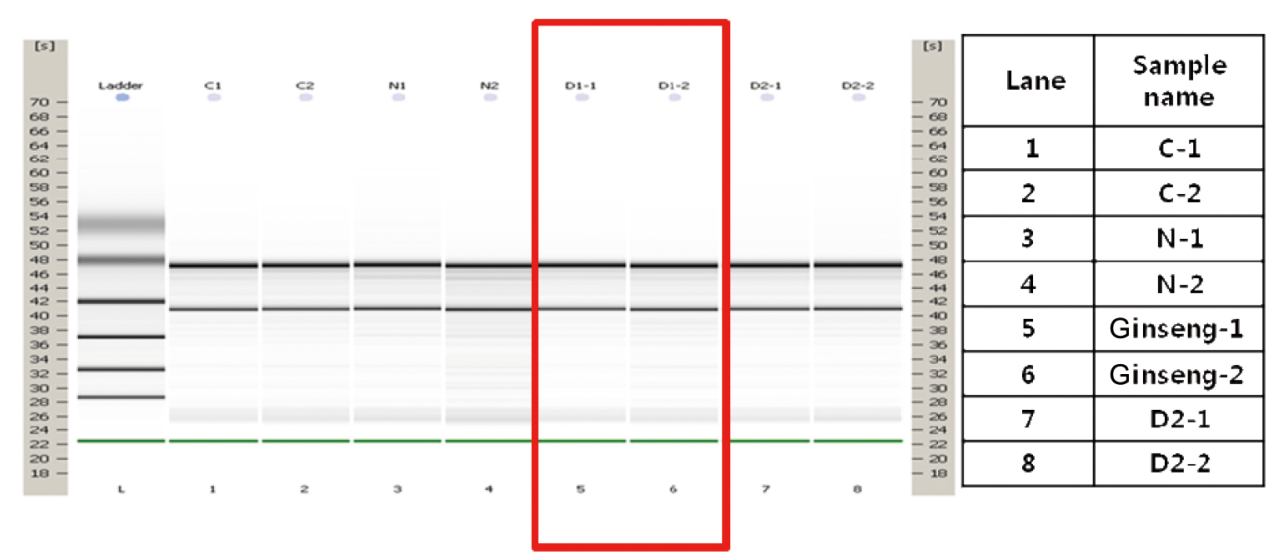

B

\begin{tabular}{|c|c|c|c|c|c|c|}
\hline Sample & $\mu \mathrm{g} / \mu \mathrm{L}$ & $\mathrm{OD}_{260 / 230}$ & Total $(\mu \mathrm{g})$ & Ratio(28s/18s) & RJN & Result \\
\hline $\mathrm{C}-1$ & 1.1536 & 2.2 & 56.5264 & 1.7 & 9.3 & Pass \\
\hline $\mathrm{C}-2$ & 0.8589 & 2.1 & 40.3730 & 1.8 & 9.0 & Pass \\
\hline $\mathrm{N}-1$ & 0.9118 & 2.0 & 42.8546 & 1.4 & 8.8 & Check \\
\hline $\mathrm{N}-2$ & 1.7089 & 2.1 & 83.7361 & 1.2 & 8.0 & Check \\
\hline Ginseng-1 & 1.4637 & 2.2 & 68.7939 & 1.8 & 9.4 & Pass \\
\hline Ginseng-2 & 1.2945 & 2.0 & 59.5470 & 1.5 & 8.7 & Check \\
\hline D2-1 & 1.4560 & 2.3 & 66.9760 & 1.8 & 9.3 & Pass \\
\hline D2-2 & 1.1279 & 2.1 & 51.8834 & 1.5 & 9.0 & Check \\
\hline
\end{tabular}

Fig. 1. RNA quality assessment. The quality of the RNA preparations was monitored using a RNA 6000 Pico Assay Solution and a 2100 Bioanalyzer (Agilent Technology). Gel-like images control and sample preparations (A) are shown. rRNA ratios (28S/18S ribosomal RNA) are shown in (B). (B) Data is from one of nine independent experiments. OD: optical density, RIN: RNA index number(1.0-10.0).

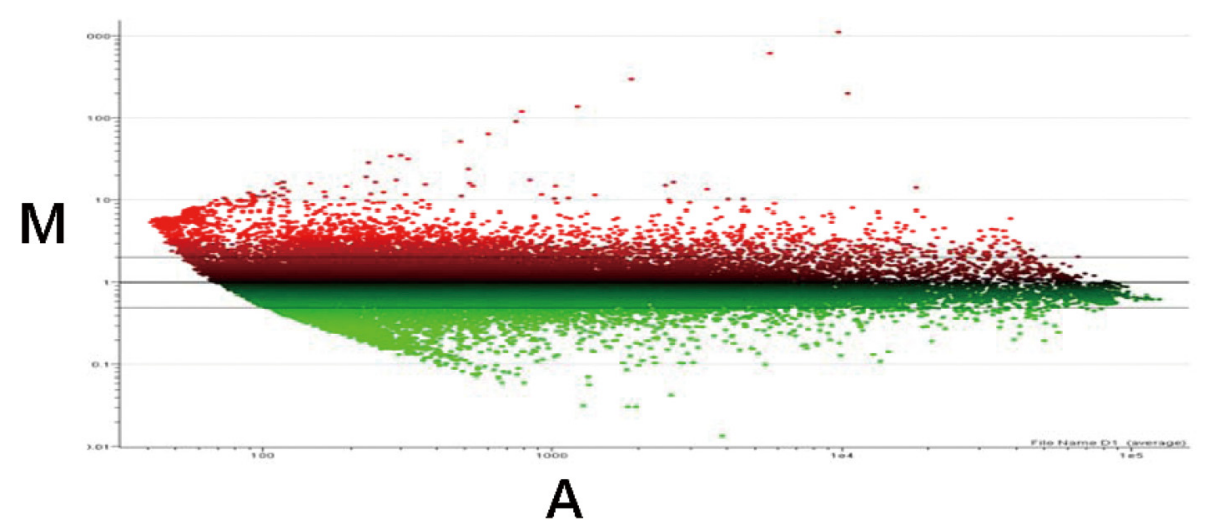

Fig. 2. The MA plot of the cDNA microarray hybridization. Gene expression signals of stressed mice and stressed mice treated with ginseng. Each point represents the average expression value for the same gene in stressed and non-stressed mice. MA plots were used to represent the $R$ and $G$ data, where $M$ (relative expression ratio)=log2(CY5/CY3). A (signal intensity)=[log2(Cy5XCy3)]/2. G is the stronger control sample (stressed mice) hybridization (CY3) and $R$ is the stronger experimental (stressed mice treated with ginseng) sample hybridization (CY5).

\section{alpha-2-HS-glycoprotein (ahsg)}

ahsg was down-regulated by $5.8 \pm 0.17$ fold. ahsg is a serum glycoprotein. $\alpha$ hsg-KO mice show impaired tolerance to ischemia suggesting that ahsg exerts a protective effect against ischemia in cardiomyocytes [16].

\section{Up-regulated immune response-related genes (Table 2) Colony stimulating factor 3 (Csf3)}

The Csf3 gene was up-regulated by 19.62 -fold. GCSF stimulates the expression of the MIP-2 receptor via STAT3-dependent transcriptional activation of Il8rb [17]. 


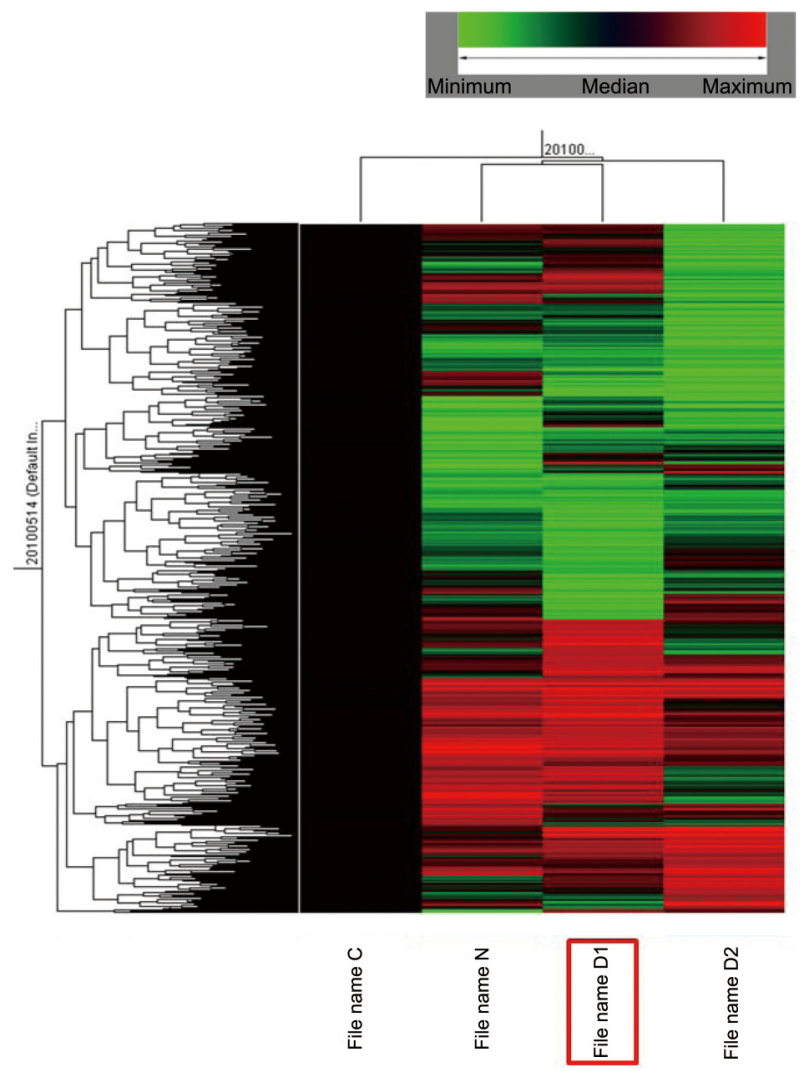

Fig. 3. Functional distribution of regulated genes. The overall distribution of the genes in each group which were up-regulated $(A)$ or down-regulated $(B)$ by a factor greater than 2-fold following ginseng extract administration on the 5th day after the immobilization challenge.

G-CSF and IL-6 provide signals that determine the angiogenic potential of resident monocytes in BM [18].

\section{C-type lectin domain family 4, member a2 (Clec4a2)}

The Clec4a2 gene was up-regulated by 5.90 -fold. This observation indicates that Dcir is a negative regulator of dendritic cell expansion and has a crucial role in maintaining the homeostasis of the immune system [19].

\section{Radical S-adenosyl methionine domain containing 2 (Rsad2)}

The Rsad 2 gene was up-regulated by 10.60 -fold. Viperin may play a central role in bacterial or parasitic infections, and may protect neutrophils and macrophages from infection [20].

\section{Up-regulated cell cycle response-related genes (Table 3) Centromere autoantigen $\mathrm{H}$ (Cenph)}

The Cenph gene was up-regulated by 14.34-fold. TRIM36 interacts with the kinetochore protein CENP-H and delays cell cycle progression [21]. Immunocytochemical analysis of CENP-I-deficient cells demonstrated that both CENP$\mathrm{I}$ and CENP-H are necessary for localization of CENP$\mathrm{C}$, but not CENP-A, to the centromere [22].

\section{Nusap1 protein (Nusap1)}

The Nusap1 gene was up-regulated by 13.26-fold. NuSAP is essential for proliferation of embryonic cells and, simultaneously, they underscore the importance of chromatin-induced spindle assembly [23].

\section{Integrin beta 1D (Itg $\beta 1)$}

The Nusap1 gene was up-regulated by 13.26 -fold. Ablation of beta1 integrin induces organ atrophy by disrupting acinar cell polarity and exposing the pancreatic parenchyma to digestive enzymes [24].

\section{Up-regulated apoptosis response-related genes (Table4)} $\mathrm{N}$-acylsphingosine amidohydrolase 2 (Asah2)

Asah2 was up-regulated by 15.87 -fold. Asah2 is an $\mathrm{N}$-acylsphingosine amidohydrolase 2. Expression of this enzyme is decreased in the small intestine in transgenic mice lacking CFTR [25]. The Asah2-encoded neutral ceramidase is a key enzyme for the catabolism of dietary sphingolipids and regulates the levels of bioactive sphingolipid metabolites in the intestinal tract [26].

\section{Fibroblast growth factor receptor 1 isoform 1 (Fgfr1)}

Fgfr1 was up-regulated by 15.64 -fold. This result indicates that MAPK/ERK activation downstream of FGFR1 is necessary for motor axon guidance, and that embryonic stem cell-derived neurons provide an important tool for dissecting intracellular pathways required for axon guidance [27]. FGFR1 is indispensable for complete differentiation and activation of osteoclasts in mice [28]. Dysfunctions in glial cells and FGF receptor signaling may therefore be implicated in neurodegenerative hearing loss associated with the normal aging process [29].

\section{Eukaryotic translation elongation factor 1 alpha 2 (Edf1a2)}

Edf1a2 was up-regulated by 15.24-fold. Eefla 2 interacts with Prdx-I to provide cells with extraordinary resistance to oxidative stress-induced cell death [30]. Expression of eEF1A-2/S1 protein is activated upon myogenic differentiation [31].

Despite these DNA microarray studies on stress response, no novel IMO stress-related genes were discovered. In this study, to search the novel genes which were up- or down-regulated by IMO stress in the mouse's adrenal cortex, the transcriptional activity of the adrenal cortex in mice challenged with days of repeated IMO 
Table 1. Immobilization stress-related changes in gene expression in the adrenal cortex

\begin{tabular}{|c|c|c|c|}
\hline GenBank ID & Fold & Gene & Function \\
\hline NM_153082 & $15.0 \pm 0.02 \uparrow$ & C330021A05Rik & DnaJ (Hsp40) homolog, subfamily C, member 27 \\
\hline NM_183027 & $14.9 \pm 0.15 \uparrow$ & Ap1s3 & Adaptor-related protein complex AP-1, sigma 3 \\
\hline $\mathrm{BC} 021475$ & $10.5 \pm 0.49 \uparrow$ & 2610301G19Rik & RIKEN cDNA 2610301G19 gene \\
\hline NM_153104 & $10.3 \pm 0.31 \uparrow$ & Phospho1 & Phosphatase, orphan 1 \\
\hline NM_145929 & $10.1 \pm 0.12 \uparrow$ & Gga1 & Golgi associated, gamma adaptin ear containing, ARF binding protein 1 \\
\hline NM_011548 & $10.0 \pm 0.04 \uparrow$ & Tcfe2a & Transcription factor E2a \\
\hline NM_009700 & $9.3 \pm 0.28 \uparrow$ & Aqp4 & Aquaporin 4 \\
\hline NM_029021 & $9.2 \pm 0.21 \uparrow$ & 4833422F24Rik & RIKEN cDNA $4833422 F 24$ gene \\
\hline NM_001031814 & $9.0 \pm 0.04 \uparrow$ & 2610207I05Rik & SMG1 homolog, phosphatidylinositol 3-kinase-related kinase (C. elegans) \\
\hline NM_009545 & $7.5 \pm 0.50 \uparrow$ & Pcgf2 & Polycomb group ring finger 2 \\
\hline NM_020587 & $7.4 \pm 0.45 \uparrow$ & Sfrs4 & Splicing factor, arginine/serine-rich 4 (SRp75) \\
\hline AK048180 & $7.2 \pm 0.17 \uparrow$ & Spata511 & Spermatogenesis associated 5-like 1 \\
\hline NM_175204 & $7.0 \pm 0.00 \uparrow$ & $5830406 J 20 R i k$ & Proteasome (prosome, macropain) subunit, beta type, 11 \\
\hline AK005867 & $6.8 \pm 0.21 \uparrow$ & 1700011H22Rik & RIKEN cDNA $1700011 \mathrm{H} 22$ gene \\
\hline AK049020 & $6.8 \pm 0.23 \uparrow$ & 9630031F12Rik & Family with sequence similarity 184 , member B \\
\hline NM_026174 & $6.6 \pm 0.37 \uparrow$ & Entpd4 & Ectonucleoside triphosphate diphosphohydrolase 4 \\
\hline NM_177730 & $6.6 \pm 0.44 \uparrow$ & Impad1 & Inositol monophosphatase domain containing 1 \\
\hline NM_011278 & $6.5 \pm 0.48 \uparrow$ & Rnf4 & Ring finger protein 4 \\
\hline NM_172758 & $6.5 \pm 0.48 \uparrow$ & ВC031853 & Solute carrier family 38 , member 7 \\
\hline NM_021295 & $6.5 \pm 0.48 \uparrow$ & Lancl1 & LanC (bacterial lantibiotic synthetase component C)-like 1 \\
\hline NM_146492 & $6.4 \pm 0.40 \uparrow$ & Olfr 724 & Olfactory receptor 724 \\
\hline NM_020255 & $6.3 \pm 0.32 \uparrow$ & Scand1 & SCAN domain-containing 1 \\
\hline NM_146683 & $6.3 \pm 0.28 \uparrow$ & Olfr1441 & Olfactory receptor 1441 \\
\hline NM_175557 & $6.3 \pm 0.27 \uparrow$ & Zfp384 & Zinc finger protein 384 \\
\hline NM_134226 & $6.3 \pm 0.26 \uparrow$ & V1rj2 & Vomeronasal 1 receptor, $\mathrm{J} 2$ \\
\hline NM_001037712 & $6.2 \pm 0.20 \uparrow$ & Kenh6 & Potassium voltage-gated channel, subfamily $\mathrm{H}$ (eag-related), member 6 \\
\hline NM_009401 & $6.2 \pm 0.16 \uparrow$ & Tnfrsf8 & Tumor necrosis factor receptor superfamily, member 8 \\
\hline NM_009392 & $6.1 \pm 0.14 \uparrow$ & Tlx2 & T-cell leukemia, homeobox 2 \\
\hline NM_146892 & $6.1 \pm 0.13 \uparrow$ & Olfr1223 & Olfactory receptor 1223 \\
\hline AK036614 & $6.1 \pm 0.08 \uparrow$ & 02-Mar & Membrane-associated ring finger $(\mathrm{C} 3 \mathrm{HC} 4) 2$ \\
\hline NM_145857 & $6.1 \pm 0.05 \uparrow$ & Nod2 & Nucleotide-binding oligomerization domain containing 2 \\
\hline NM_001010941 & $6.0 \pm 0.01 \uparrow$ & Gpr12 & G-protein coupled receptor 12 \\
\hline NM_013855 & $5.9 \pm 0.07 \uparrow$ & Abca3 & ATP-binding cassette, sub-family A ( $\mathrm{ABC} 1$ ), member 3 \\
\hline NM_030725 & $5.8 \pm 0.19 \uparrow$ & Syt13 & Synaptotagmin XIII \\
\hline NM_198621 & $5.8 \pm 0.21 \uparrow$ & $\mathrm{Gm} 443$ & Predicted gene 443 \\
\hline NM_010438 & $5.6 \pm 0.38 \uparrow$ & Hk1 & Hexokinase 1 \\
\hline NM_011348 & $5.6 \pm 0.38 \uparrow$ & Sema3e & Sema domain, immunoglobulin domain (Ig), short basic domain, secreted, (semaphorin) 3E \\
\hline NM_021535 & $5.6 \pm 0.39 \uparrow$ & Smu1 & Smu-1 suppressor of mec- 8 and unc-52 homolog (C. elegans) \\
\hline NM_007577 & $5.6 \pm 0.40 \uparrow$ & C5ar1 & Complement component 5 a receptor 1 \\
\hline NM_001011820 & $5.4 \pm 0.40 \uparrow$ & Olfr1359 & Olfactory receptor 1359 \\
\hline NM_012028 & $5.4 \pm 0.39 \uparrow$ & St6galnac5 & $\begin{array}{l}\text { ST6 (alpha- } \mathrm{N} \text {-acetyl-neuraminyl-2,3-beta-galactosyl-1,3)-N-acetylgalactosaminide alpha-2,6- } \\
\text { sialyltransferase } 5\end{array}$ \\
\hline NM_027427 & $5.3 \pm 0.35 \uparrow$ & Taf15 & TAF15 RNA polymerase II, TATA box binding protein (TBP)-associated factor \\
\hline NM_207685 & $5.3 \pm 0.34 \uparrow$ & Elavl2 & ELAV (embryonic lethal, abnormal vision, Drosophila)-like 2 (Hu antigen B) \\
\hline NM_175263 & $5.3 \pm 0.33 \uparrow$ & 5730593N15Rik & Notum pectinacetylesterase homolog (Drosophila) \\
\hline
\end{tabular}


Table 1. Continued-1

\begin{tabular}{|c|c|c|c|}
\hline GenBank ID & Fold & Gene & Function \\
\hline NM_029607 & $5.3 \pm 0.28 \uparrow$ & 2310003C23Rik & RIKEN cDNA 2310003C23 gene \\
\hline NM_146206 & $5.3 \pm 0.26 \uparrow$ & Tpcn2 & Two pore segment channel 2 \\
\hline NM_023117 & $5.2 \pm 0.20 \uparrow$ & $\mathrm{Cdc} 25 \mathrm{~b}$ & Cell division cycle 25 homolog B (S. pombe) \\
\hline NM_053185 & $5.2 \pm 0.19 \uparrow$ & Col4a6 & Collagen, type IV, alpha 6 \\
\hline NM_026976 & $5.2 \pm 0.17 \uparrow$ & Faim3 & Fas apoptotic inhibitory molecule 3 \\
\hline AK042979 & $5.2 \pm 0.16 \uparrow$ & Tbc1d4 & TBC1 domain family, member 4 \\
\hline NM_146709 & $5.2 \pm 0.16 \uparrow$ & Olfr411 & Olfactory receptor 411 \\
\hline NM_019826 & $5.1 \pm 0.11 \uparrow$ & Ivd & Isovaleryl coenzyme A dehydrogenase \\
\hline NM_029090 & $5.1 \pm 0.09 \uparrow$ & 1200013P24Rik & N-acetyltransferase 15 (GCN5-related, putative) \\
\hline NM_032397 & $5.0 \pm 0.04 \uparrow$ & Kcnn1 & Potassium intermediate/small conductance calcium-activated channel, subfamily $\mathrm{N}$, member 1 \\
\hline NM_172641 & $5.0 \pm 0.03 \uparrow$ & 9930023K05Rik & RIKEN cDNA 9930023K05 gene \\
\hline NM_183148 & $5.0 \pm 0.02 \uparrow$ & C79267 & Intermediate filament family orphan 2 \\
\hline NM_027100 & $4.9 \pm 0.06 \uparrow$ & Rwdd2 & RWD domain containing $2 \mathrm{~A}$ \\
\hline NM_178666 & $4.9 \pm 0.06 \uparrow$ & E430004N04Rik & Thymocyte selection pathway associated \\
\hline NM_029001 & $4.9 \pm 0.06 \uparrow$ & Elov17 & ELOVL family member 7, elongation of long chain fatty acids (yeast) \\
\hline NM_019553 & $4.9 \pm 0.13 \uparrow$ & $\operatorname{Ddx} 21$ & DEAD (Asp-Glu-Ala-Asp) box polypeptide 21 \\
\hline NM_008267 & $4.8 \pm 0.16 \uparrow$ & Hoxb13 & Homeo box B13 \\
\hline NM_026668 & $4.8 \pm 0.22 \uparrow$ & 4930558O21Rik & Leucine-rich repeats and IQ motif containing 4 \\
\hline NM_147069 & $4.8 \pm 0.23 \uparrow$ & Olfr686 & Olfactory receptor 686 \\
\hline NM_198614 & $4.7 \pm 0.28 \uparrow$ & 4932409I22Rik & Family with sequence similarity 148 , member C \\
\hline NM_001039169 & $4.7 \pm 0.30 \uparrow$ & Eif4e 2 & Eukaryotic translation initiation factor $4 \mathrm{E}$ member 2 \\
\hline NM_026470 & $4.7 \pm 0.31 \uparrow$ & Spata6 & Spermatogenesis associated 6 \\
\hline NM_146653 & $4.7 \pm 0.32 \uparrow$ & Olfr435 & Olfactory receptor 435 \\
\hline BC048580 & $4.7 \pm 0.32 \uparrow$ & Brpf3 & Bromodomain and PHD finger containing, 3 \\
\hline NM_177164 & $4.7 \pm 0.33 \uparrow$ & A830006F12Rik & RIKEN cDNA A830006F12 gene \\
\hline AK084222 & $4.7 \pm 0.34 \uparrow$ & 2700094F01Rik & Transmembrane protein 209 \\
\hline NM_178773 & $4.7 \pm 0.35 \uparrow$ & Tmem16d & Anoctamin 4 \\
\hline NM_029413 & $4.6 \pm 0.37 \uparrow$ & Morc4 & Microrchidia 4 \\
\hline NM_031376 & $4.6 \pm 0.37 \uparrow$ & Pik3ap1 & Phosphoinositide-3-kinase adaptor protein 1 \\
\hline XM_975192 & $4.6 \pm 0.39 \uparrow$ & Camsap1 & Calmodulin regulated spectrin-associated protein 1 \\
\hline NM_183316 & $4.6 \pm 0.41 \uparrow$ & Snapc5 & Small nuclear RNA activating complex, polypeptide 5 \\
\hline NM_022320 & $4.6 \pm 0.41 \uparrow$ & Gpr35 & G protein-coupled receptor 35 \\
\hline AK045799 & $4.6 \pm 0.43 \uparrow$ & Fhad1 & Forkhead-associated (FHA) phosphopeptide binding domain 1 \\
\hline XM_888058 & $4.6 \pm 0.44 \uparrow$ & C330016K18Rik & Solute carrier family 4 , sodium bicarbonate cotransporter, member 5 \\
\hline NM_009233 & $4.5 \pm 0.47 \uparrow$ & Sox 1 & SRY-box containing gene 1 \\
\hline NM_001013823 & $4.5 \pm 0.49 \uparrow$ & LOC435285 & Keratin associated protein $4-16$ \\
\hline NM_175445 & $4.5 \pm 0.48 \uparrow$ & Rassf2 & Ras association (RalGDS/AF-6) domain family member 2 \\
\hline NM_028065 & $4.5 \pm 0.48 \uparrow$ & Tnrc5 & Canopy 3 homolog (zebrafish) \\
\hline NM_080452 & $4.5 \pm 0.48 \uparrow$ & Mrps2 & Mitochondrial ribosomal protein S2 \\
\hline NM_147109 & $4.5 \pm 0.47 \uparrow$ & Olfr577 & Olfactory receptor 577 \\
\hline NM_010746 & $4.5 \pm 0.45 \uparrow$ & Ncr1 & Natural cytotoxicity triggering receptor 1 \\
\hline NM_134249 & $4.5 \pm 0.45 \uparrow$ & Timd2 & T-cell immunoglobulin and mucin domain containing 2 \\
\hline NM_008470 & $4.4 \pm 0.41 \uparrow$ & Krt16 & Keratin 16 \\
\hline NM_001038590 & $4.4 \pm 0.38 \uparrow$ & Cldn19 & Claudin 19 \\
\hline
\end{tabular}


Table 1. Contined-2

\begin{tabular}{|c|c|c|c|}
\hline GenBank ID & Fold & Gene & Function \\
\hline NM_145388 & $4.4 \pm 0.38 \uparrow$ & Best2 & Bestrophin 2 \\
\hline NM_025834 & $4.4 \pm 0.37 \uparrow$ & Proz & Protein Z, vitamin K-dependent plasma glycoprotein \\
\hline XM_619828 & $4.4 \pm 0.36 \uparrow$ & Scrt2 & Scratch homolog 2, zinc finger protein (Drosophila) \\
\hline AK145379 & $4.4 \pm 0.36 \uparrow$ & H19 & H19 fetal liver mRNA \\
\hline NM_011487 & $4.3 \pm 0.35 \uparrow$ & Stat4 & Signal transducer and activator of transcription 4 \\
\hline NM_001039365 & $4.3 \pm 0.32 \uparrow$ & Mobp & Myelin-associated oligodendrocytic basic protein \\
\hline NM_008865 & $4.3 \pm 0.30 \uparrow$ & Csh2 & Prolactin family 3 , subfamily b, member 1 \\
\hline NM_019940 & $4.3 \pm 0.29 \uparrow$ & Zfp111 & Zinc finger protein 111 \\
\hline NM_010085 & $4.3 \pm 0.27 \uparrow$ & Adam26a & A disintegrin and metallopeptidase domain 26A (testase 3 ) \\
\hline AK002926 & $4.3 \pm 0.25 \uparrow$ & Klf13 & Kruppel-like factor 13 \\
\hline AK044798 & $4.2 \pm 0.25 \uparrow$ & $\operatorname{Atg} 4 d$ & Autophagy-related 4D (yeast) \\
\hline NM_029781 & $4.2 \pm 0.24 \uparrow$ & Rab36 & RAB36, member RAS oncogene family \\
\hline NM_153805 & $4.2 \pm 0.24 \uparrow$ & Pkn3 & Protein kinase N3 \\
\hline NM_147110 & $4.2 \pm 0.23 \uparrow$ & Olfr570 & Olfactory receptor 570 \\
\hline NM_020287 & $4.2 \pm 0.21 \uparrow$ & Insm2 & Insulinoma-associated 2 \\
\hline NM_027069 & $4.2 \pm 0.21 \uparrow$ & 1700018F24Rik & RIKEN cDNA 1700018F24 gene \\
\hline AK008094 & $4.2 \pm 0.17 \uparrow$ & Igl-V1 & Immunoglobulin lambda chain, variable 1 \\
\hline NM_182636 & $4.2 \pm 0.17 \uparrow$ & $\mathrm{BC} 052055$ & von Willebrand factor A domain containing 5B2 \\
\hline NM_010828 & $4.2 \pm 0.17 \uparrow$ & Cited2 & $\mathrm{Cbp} / \mathrm{p} 300$-interacting transactivator, with Glu/Asp-rich carboxy-terminal domain, 2 \\
\hline NM_199313 & $4.2 \pm 0.17 \uparrow$ & AY053573 & Retinol dehydrogenase 18 \\
\hline NM_139225 & $4.2 \pm 0.16 \uparrow$ & Defb10 & Defensin beta 10 \\
\hline AK033743 & $4.1 \pm 0.10 \uparrow$ & Len6 & Lipocalin 6 \\
\hline NM_172896 & $4.1 \pm 0.09 \uparrow$ & Nalp4a & NLR family, pyrin domain containing 4A \\
\hline NM_010407 & $4.1 \pm 0.07 \uparrow$ & Hck & Hemopoietic cell kinase \\
\hline NM_026893 & $4.1 \pm 0.07 \uparrow$ & Wdr40a & WD repeat domain $40 \mathrm{~A}$ \\
\hline NM_175225 & $4.1 \pm 0.05 \uparrow$ & Tasp1 & Taspase, threonine aspartase 1 \\
\hline NM_009567 & $4.0 \pm 0.05 \uparrow$ & Zfp93 & Zinc finger protein 93 \\
\hline NM_139296 & $4.0 \pm 0.04 \uparrow$ & $\operatorname{Moxd} 2$ & Monooxygenase, DBH-like 2 \\
\hline NM_008498 & $4.0 \pm 0.01 \uparrow$ & Lhx 1 & LIM homeobox protein 1 \\
\hline NM_173405 & $4.0 \pm 0.01 \uparrow$ & 6530401C20Rik & Archaelysin family metallopeptidase 1 \\
\hline NM_138630 & $4.0 \pm 0.00 \uparrow$ & Arhgap4 & Rho GTPase activating protein 4 \\
\hline NM_008577 & $4.1 \pm 0.24 \downarrow$ & $\mathrm{S} 1 \mathrm{c} 3 \mathrm{a} 2$ & Solute carrier family 3 (activators of dibasic and neutral amino acid transport), member 2 \\
\hline NM_007981 & $4.2 \pm 0.24 \downarrow$ & Acs11 & Acyl-CoA synthetase long-chain family member 1 \\
\hline NM_008645 & $4.2 \pm 0.24 \downarrow$ & Mug1 & Murinoglobulin 1 \\
\hline NM_031188 & $4.2 \pm 0.24 \downarrow$ & Mup1 & Major urinary protein 1 \\
\hline NM_008407 & $4.2 \pm 0.24 \downarrow$ & Itih3 & Inter-alpha trypsin inhibitor, heavy chain 3 \\
\hline NM_019423 & $4.2 \pm 0.24 \downarrow$ & Elov12 & Elongation of very long chain fatty acids (FEN1/Elo2, SUR4/Elo3, yeast)-like 2 \\
\hline NM_009654 & $4.2 \pm 0.24 \downarrow$ & Alb1 & Albumin \\
\hline NM_080434 & $4.4 \pm 0.23 \downarrow$ & Apoa5 & Apolipoprotein A-V \\
\hline NM_013820 & $4.4 \pm 0.23 \downarrow$ & $\mathrm{Hk} 2$ & Hexokinase 2 \\
\hline $\mathrm{BC} 037152$ & $4.4 \pm 0.23 \downarrow$ & Mup1 & Major urinary protein 1 \\
\hline NM_054094 & $4.4 \pm 0.23 \downarrow$ & Acsm1 & Acyl-CoA synthetase medium-chain family member 1 \\
\hline NM_009876 & $4.5 \pm 0.22 \downarrow$ & Cdkn1c & Cyclin-dependent kinase inhibitor 1C (P57) \\
\hline NM_008646 & $4.5 \pm 0.22 \downarrow$ & Mug2 & $\mathrm{C} 3$ and PZP-like, alpha-2-macroglobulin domain containing 8 \\
\hline
\end{tabular}


Table 1. Contined-3

\begin{tabular}{|c|c|c|c|}
\hline GenBank ID & Fold & Gene & Function \\
\hline NM_010006 & $4.5 \pm 0.22 \downarrow$ & Cyp2d9 & Cytochrome P450, family 2, subfamily d, polypeptide 9 \\
\hline NM_010559 & $5.0 \pm 0.20 \downarrow$ & Il6ra & Interleukin 6 receptor, alpha \\
\hline NM_146148 & $5.0 \pm 0.20 \downarrow$ & C8a & Complement component 8 , alpha polypeptide \\
\hline NM_174870 & $5.0 \pm 0.20 \downarrow$ & Slc26a1 & Solute carrier family 26 (sulfate transporter), member 1 \\
\hline NM_144511 & $5.1 \pm 0.20 \downarrow$ & EG13909 & Esterase 31 \\
\hline NM_008986 & $5.1 \pm 0.19 \downarrow$ & Ptrf & Polymerase I and transcript release factor \\
\hline NM_145356 & $5.2 \pm 0.19 \downarrow$ & Zbtb7c & Zinc finger and BTB domain containing $7 \mathrm{C}$ \\
\hline NM_007817 & $5.2 \pm 0.19 \downarrow$ & Cyp2f2 & Cytochrome P450, family 2, subfamily f, polypeptide 2 \\
\hline NM_011315 & $5.2 \pm 0.19 \downarrow$ & Saa3 & Serum amyloid A 3 \\
\hline NM_008819 & $5.2 \pm 0.19 \downarrow$ & Pemt & Phosphatidylethanolamine N-methyltransferase \\
\hline AK028683 & $5.4 \pm 0.18 \downarrow$ & Cps1 & Carbamoyl-phosphate synthetase 1 \\
\hline NM_023114 & $5.4 \pm 0.18 \downarrow$ & Apoc3 & Apolipoprotein C-III \\
\hline NM_017399 & $5.5 \pm 0.18 \downarrow$ & Fabp1 & Fatty acid binding protein 1 , liver \\
\hline NM_144903 & $5.7 \pm 0.18 \downarrow$ & Aldob & Aldolase B, fructose-bisphosphate \\
\hline NM_010005 & $5.8 \pm 0.17 \downarrow$ & Cyp2d10 & Cytochrome P450, family 2, subfamily d, polypeptide 10 \\
\hline NM_013465 & $5.8 \pm 0.17 \downarrow$ & Ahsg & Alpha-2-HS-glycoprotein \\
\hline NM_010210 & $5.8 \pm 0.17 \downarrow$ & Fhit & Fragile histidine triad gene \\
\hline NM_019792 & $6.1 \pm 0.16 \downarrow$ & Cyp3a25 & Cytochrome P450, family 3, subfamily a, polypeptide 25 \\
\hline NM_010004 & $6.1 \pm 0.16 \downarrow$ & Cyp2c40 & Cytochrome P450, family 2 , subfamily c, polypeptide 40 \\
\hline NM_144903 & $6.2 \pm 0.16 \downarrow$ & Aldob & Aldolase B, fructose-bisphosphate \\
\hline NM_029821 & $6.3 \pm 0.16 \downarrow$ & 1190003J15Rik & RIKEN cDNA 1190003 J15 gene \\
\hline NM_011707 & $6.3 \pm 0.16 \downarrow$ & Vtn & Vitronectin \\
\hline NM_023114 & $6.3 \pm 0.16 \downarrow$ & Apoc3 & Apolipoprotein C-III \\
\hline NM_198615 & $6.4 \pm 0.16 \downarrow$ & Rkhd1 & Mex3 homolog D (C. elegans) \\
\hline NM_011314 & $6.6 \pm 0.15 \downarrow$ & Saa2 & Serum amyloid A 2 \\
\hline AK082385 & $7.3 \pm 0.14 \downarrow$ & Indol1 & Indoleamine 2,3-dioxygenase 2 \\
\hline NM_017371 & $7.5 \pm 0.13 \downarrow$ & Hpxn & Hemopexin \\
\hline NM_011458 & $7.8 \pm 0.13 \downarrow$ & Serpina3k & Serine (or cysteine) peptidase inhibitor, clade A, member $3 \mathrm{~K}$ \\
\hline NM_011316 & $8.1 \pm 0.12 \downarrow$ & Saa4 & Serum amyloid A 4 \\
\hline NM_011458 & $9.1 \pm 0.11 \downarrow$ & Serpina $3 \mathrm{k}$ & Serine (or cysteine) peptidase inhibitor, clade A, member $3 \mathrm{~K}$ \\
\hline NM_007870 & $9.2 \pm 0.11 \downarrow$ & Dnase113 & Deoxyribonuclease 1-like 3 \\
\hline NM_017473 & $9.4 \pm 0.11 \downarrow$ & $\operatorname{Rdh} 7$ & Retinol dehydrogenase 7 \\
\hline BC037008 & $9.6 \pm 0.10 \downarrow$ & Serpinalb & Serine (or cysteine) preptidase inhibitor, clade $\mathrm{A}$, member $1 \mathrm{~B}$ \\
\hline NM_009245 & $9.7 \pm 0.10 \downarrow$ & Serpina1c & Serine (or cysteine) peptidase inhibitor, clade A, member 1C \\
\hline NM_009512 & $9.8 \pm 0.10 \downarrow$ & Slc27a5 & Solute carrier family 27 (fatty acid transporter), member 5 \\
\hline NM_010168 & $10.0 \pm 0.10 \downarrow$ & $\mathrm{F} 2$ & Coagulation factor II \\
\hline
\end{tabular}

$\uparrow$ and $\downarrow$ indicate the up- and down-regulation of gene expression. $4.0 \downarrow$ means that the expression level was decreased to $1 / 4$.

was quantitatively analyzed using cDNA microarray. After sacrificing the mice on the last day of the IMO stress challenge, the adrenal cortex was quickly separated from the adrenal gland and used for the purification of total RNA. The significantly up- or down-regulated genes were identified using the following criteria: significant difference between the control and IMO groups, that is, $p<0.05$ ( $t$-test) and a mean fold-change $>1.5$. A fluctuation in gene expression levels over 4-fold was considered to be sufficient to induce significant biochemical 
Table 2. Immune response-related genes

\begin{tabular}{|c|c|c|c|}
\hline GenBank ID & Fold & Gene & Function \\
\hline A_51_P317176 & 19.62196 & Csf3 & Colony stimulating factor 3 (granulocyte) \\
\hline A_52_P356106 & 15.894692 & $\mathrm{Clec} 4 \mathrm{a} 2$ & C-type lectin domain family 4 , member a2 C-type lectin domain family 4 , member a2 \\
\hline A_51_P505134 & 10.594688 & Rsad2 & Radical S-adenosyl methionine domain containing 2 \\
\hline A_52_P560113 & 10.428145 & $\mathrm{H} 2-\mathrm{B} 1$ & Histocompatibility 2 , blastocyst \\
\hline A_52_P651316 & 8.93424 & $\mathrm{Cd} 79 \mathrm{a}$ & CD79A antigen (immunoglobulin-associated alpha) \\
\hline A_51_P166941 & 8.156842 & Irak3 & Interleukin-1 receptor-associated kinase 3 \\
\hline A_52_P66520 & 7.9745355 & Clec12a & C-type lectin domain family 12 , member a \\
\hline A_52_P100926 & 7.9733095 & Illa & Interleukin 1 alpha \\
\hline A_51_P168115 & 7.8946457 & Il21 & Interleukin 21 \\
\hline A_51_P335945 & 7.8921895 & Pag1 & Phosphoprotein associated with glycosphingolipid microdomains 1 \\
\hline A_51_P512085 & 7.79134 & Colec 12 & Collectin sub-family member 12 \\
\hline A_52_P641747 & 7.7182136 & Sh2d1a & $\mathrm{SH} 2$ domain protein $1 \mathrm{~A}$ \\
\hline A_51_P464703 & 7.5314245 & $\mathrm{Ccl} 8$ & Chemokine (C-C motif) ligand 8 \\
\hline A_51_P328537 & 7.4499946 & Eda & Ectodysplasin-A \\
\hline A_52_P523946 & 7.4396567 & $\operatorname{Ddx} 58$ & DEAD (Asp-Glu-Ala-Asp) box polypeptide 58 \\
\hline A_51_P436652 & 7.33316 & $\mathrm{Ccl} 7$ & Chemokine (C-C motif) ligand 7 \\
\hline
\end{tabular}

Table 3. Cell cycle-related genes

\begin{tabular}{clll}
\hline GenBank ID & \multicolumn{1}{c}{ Fold } & Gene & \multicolumn{1}{c}{ Function } \\
\hline A_51_P404766 & 14.9005575 & Tlk2 & \\
A_51_P492830 & 14.337363 & Cenph & Centromere autoantigen H \\
A_52_P69281 & 13.263976 & Nusap1 & Nusap1 protein \\
A_51_P302527 & 12.605151 & Nek1 & \\
A_52_P69236 & 11.924297 & Itgb1 & Integrin beta1D \\
A_51_P340003 & 11.358889 & Sycp1 & Synaptonemal complex protein 1 \\
A_51_P419169 & 10.672192 & Suv39h2 & Suppressor of variegation 3-9 homologue 2 \\
A_52_P615877 & 10.460364 & Cdc25b & Cell division cycle 25 homolog B \\
A_52_P259250 & 9.951616 & Evi5 & Ecotropic viral integration site 5 \\
A_52_P131891 & 9.72852 & Spag5 & Sperm associated antigen 5 \\
A_52_P512955 & 9.418641 & Anln & Anillin \\
A_51_P475523 & 9.060346 & Brca1 & Breast cancer 1 \\
A_52_P417489 & 8.984837 & Cdk6 & Cyclin-dependent kinase 6 \\
A_52_P513394 & 8.50183 & Brca2 & Breast cancer 2 \\
A_52_P404570 & 8.478805 & Dbf4 & Dbf4 protein \\
A_51_P369056 & 8.123366 & Prox1 & Prospero-related homeobox 1 \\
\hline
\end{tabular}

and physiological changes in living cells. By using these criteria, 176 genes out of 41,174 genes were selected since their expression levels were significantly modulated in adrenal cortex by stress. 118 genes were up-regulated by IMO stress while 49 genes were down-regulated. Genes related to immune responses, apoptosis, and signal transduction were activated by a factor of 1.5 to 15.0 (Table 2). The expression of specific disease-related genes, such as Aqp4 (spinal cord injury), was strongly stimulated by stress. Among the up- regulated genes, nine olfactory receptors including Olfr 724, Olfr 1441, Olfr 1223, Olfr 1359, Olfr 411, Olfr 686, Olfr 435, Olfr 577, and Olfr 570 were highly overexpressed. mRNA processing genes, such as splicing factor, arginine/serine-rich 4 (Sfrs4), was also up-regulated over 7.4-fold. A majority of the up-regulated genes were found to be involved in transcriptional regulation (Tcfe2a, Stat4), or were cell signaling molecules (Camsap1) and receptors (V1ri2, Tnfrsf8, Gpr12, C5ar1, Gpr35, and Ncr1). 
Table 4. Apoptosis-related genes

\begin{tabular}{llll}
\hline GenBank ID & \multicolumn{1}{c}{ Fold } & Gene & \multicolumn{1}{c}{ Function } \\
\hline A_52_P56792 & 15.868544 & Asah2 & N-acylsphingosine amidohydrolase 2 \\
A_52_P552062 & 15.643426 & Fgfr1 & Fibroblast growth factor receptor 1 isoform 1 \\
A_51_P485862 & 15.239337 & Eef1a2 & Eukaryotic translation elongation factor 1 alpha 2 \\
A_52_P401473 & 14.617006 & Ntn1 & \\
A_52_P403582 & 13.489419 & Trim39 & Tripartite motif protein 39 \\
A_52_P131353 & 9.06976 & Camk1d & Calcium/calmodulin-dependent protein kinase 1D \\
A_51_P475523 & 9.060346 & Brca1 & Breast cancer 1 \\
A_52_P513394 & 8.50183 & Brca2 & Breast cancer 2 \\
A_52_P282110 & 8.444962 & Mef2c & \\
A_51_P484880 & 8.224829 & Bc12111 & BCL2-like 11 apoptosis facilitator isoform 1 \\
A_51_P166941 & 8.156842 & Irak3 & Interleukin-1 receptor-associated kinase 3 \\
A_52_P204710 & 8.133725 & Sgk3 & \\
A_51_P173285 & 7.8557196 & Nkx2-5 & NK2 transcription factor related, locus 5 \\
A_51_P216550 & 7.837571 & Trp63 & Transformation related protein 63 \\
A_51_P248935 & 7.737255 & Terc & \\
A_52_P330424 & 7.6651587 & Il2ra & \\
\hline
\end{tabular}

It was reported that restraint stress often modulates the immune system by either immunoactivation or immunosuppression depending on the intrinsic properties of the stress. Acute stress basically enhances the immune system to protect the body from stress-related dysfunction, whereas chronic stress gradually reduces overall immunity by inducing apoptotic death of immune cells and suppressing the basal expression of survival genes such as tumor suppressors, chaperones, and neurotrophin. In order to verify the gene expression levels obtained from the micro- array analysis, six genes were selected for mRNA expression profiling by RT-PCR. Values from RT-PCR analyses are expressed as the mean and standard deviation from at least three independent experiments. The expression levels of five of these genes were found to decrease, but in case of phospho 1 the expression increased, which concurred with the microarray experiment results (Fig. 4).

The effects of IMO stress on the six genes demonstrated by RT-PCR are shown in Table 5. There were significant decreases in the cortical mRNA expression of

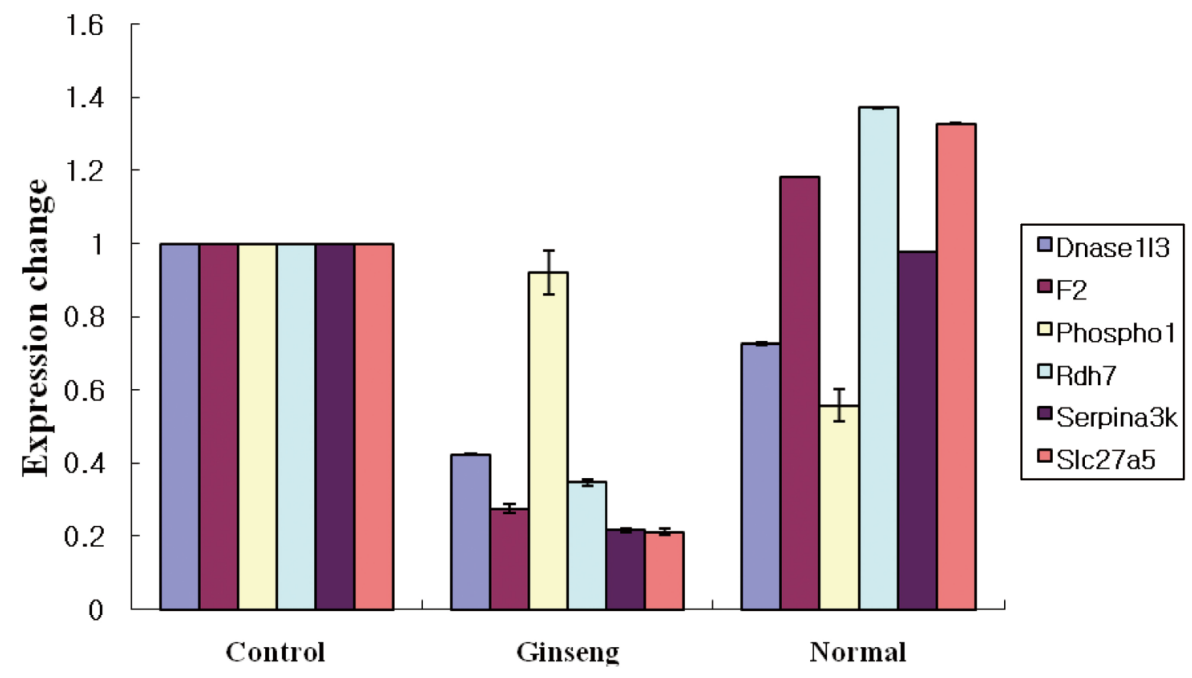

Fig. 4. Real-time polymerase chain reaction $(P C R)$ analyses of immobilization stress-related genes in the adrenal cortex. The expression level of each target PCR product was normalized to that of glyceraldehyde-3-phosphate dehydrogenase, a house-keeping gene. Values are means and standard deviations from at least three independent experiments. 
Table 5. Sequences of the primers used for real-time polymerase chain reaction

\begin{tabular}{lllc}
\hline Gene name & \multicolumn{1}{c}{ Forward primer sequence } & \multicolumn{1}{c}{ Reverse primer sequence } & Size (bp) \\
\hline Serpina3k & AAG GAA GTC TTC ACC GAA CAA G & GCC TTA CGA ATG CCA CCA ATA AC & 155 \\
Dnase113 & GAG TGT TTG CTC CCT TCC TG & GGT TGC TTC TGA GAC TGG GA & 193 \\
F2 & CAA CAT GTT CTG TGC TGG CT & TAC CAG CGG TTG TTA AAG GG & 111 \\
Slc27a5 & GCC TAT GCC ACA CCT CAT TT & GGT TCC TTC ACA CAC AGC CT & 198 \\
Phospho1 & GCT GTC TAC GAG ACC ATC CC & GAG CCC TGT TTG GCT ATG AA & 71 \\
Rdh7 & CAC CTA GGA TGA GCT TTG CC & AAC GGC ATT AGG ATG TGG AG & 150 \\
\hline
\end{tabular}

five out of the six genes (Serpina 3K, Dnase113, F2, S1c27a5, and Rdh7) after the mice were subjected to IMO stress for $1 \mathrm{~h}$. However, the cortical mRNA expression of phospho 1 was significantly increased in. The phosphol gene is highly expressed in bone tissue, and phospho 1 protein localization is restricted to sites of skeletal mineralization in the mouse. Furthermore, phosphol protein is cytosolic and active within murine osteoblasts [32,33]. Thrombin plays a major role in extra-intestinal thrombus formation associated with experimentally-induced colitis. It also appears to contribute to the propagation/stabilization, rather than initiation, phase of colitis-associated thrombogenesis at distant vascular sites [34]. We also identified several up-regulated genes which have not been reported to be associated with stress response, including Caudin, and Tlx2, and have been known to be harmful to cells at high expression levels. Furthermore, Aqp4 and Defb10 could be recommended as novel key elements that mediated the stress response. These might be used as new targets for the development of therapeutics for treating chronic stress or depression although further studies of the genetic and structural aspects of each target are required.

\section{ACKNOWLEDGEMENTS}

This study was financially supported by the Medicinal Crops Division, Ginseng and Medicinal Plants Research Institute Rural Development Administration (20070201030031).

\section{REFERENCES}

1. Padgett DA, Glaser R. How stress influences the immune response. Trends Immunol 2003;24:444-448.

2. Cannon WB. Organization for physiological homeostasis. Physiol Rev 1929;9:399-431.

3. Pacak K, Palkovits M. Stressor specificity of central neuroendocrine responses: implications for stress-related disorders. Endocr Rev 2001;22:502-548.

4. Yun SJ, Hahm DH, Lee EH. Immobilization stress induces the expression of alphaB-crystallin in rat hippocampus: implications of glial activation in stress-mediated hippocampal degeneration. Neurosci Lett 2002;324:45-48.

5. Rage F, Givalois L, Marmigere F, Tapia-Arancibia L, Arancibia S. Immobilization stress rapidly modulates BDNF mRNA expression in the hypothalamus of adult male rats. Neuroscience 2002;112:309-318.

6. Choi EH, Lee HJ, Kim CJ, Kim JT, Kwun IS, Kim Y. Anti-stress effects of ginseng in immobilization-stressed rats. J Food Sci Nutr 2004;9:253-258.

7. Dang H, Chen Y, Liu X, Wang Q, Wang L, Jia W, Wang Y. Antidepressant effects of ginseng total saponins in the forced swimming test and chronic mild stress models of depression. Prog Neuropsychopharmacol Biol Psychiatry 2009;33:1417-1424.

8. Cho JG, Lee MK, Lee JW, Park HJ, Lee DY, Lee YH, Yang DC, Baek NI. Physicochemical characterization and NMR assignments of ginsenosides Rb1, Rb2, Rc, and Rd isolated from Panax ginseng. J Ginseng Res 2010;34:113121.

9. Lee SH, Jung BH, Kim SY, Lee EH, Chung BC. The antistress effect of ginseng total saponin and ginsenoside $\mathrm{Rg} 3$ and Rb1 evaluated by brain polyamine level under immobilization stress. Pharmacol Res 2006;54:46-49.

10. Renaud S, Hays AP, Brannagan TH 3rd, Sander HW, Edgar M, Weimer LH, Olarte MR, Dalakas MC, Xiang Z, Danon $\mathrm{MJ}$, et al. Gene expression profiling in chronic inflammatory demyelinating polyneuropathy. J Neuroimmunol 2005;159:203-214.

11. Williams NM, O’Donovan MC, Owen MJ. Genome scans and microarrays: converging on genes for schizophrenia? $\mathrm{Ge}-$ nome Biol 2002;3:REVIEWS1011.

12. Lee HC, Chang DE, Yeom M, Kim GH, Choi KD, Shim I, Lee HJ, Hahm DH. Gene expression profiling in hypothalamus of immobilization-stressed mouse using cDNA microarray. Brain Res Mol Brain Res 2005;135:293-300.

13. Dudoit S, Yang YH, Callow MJ, Speed TP. Statistical methods for identifying differentially expressed genes in replicated cDNA microarray experiments. Stat Sin 2002;12:111-140.

14. Kaiser V, Diamond G. Expression of mammalian defensin genes. J Leukoc Biol 2000;68:779-784. 
15. Niyonsaba F, Nagaoka I, Ogawa H. Human defensins and cathelicidins in the skin: beyond direct antimicrobial properties. Crit Rev Immunol 2006;26:545-576.

16. Ren J, Davidoff AJ. alpha2-Heremans Schmid glycoprotein, a putative inhibitor of tyrosine kinase, prevents glucose toxicity associated with cardiomyocyte dysfunction. Diabetes Metab Res Rev 2002;18:305-310.

17. Nguyen-Jackson H, Panopoulos AD, Zhang H, Li HS, Watowich SS. STAT3 controls the neutrophil migratory response to CXCR2 ligands by direct activation of G-CSF-induced CXCR2 expression and via modulation of CXCR2 signal transduction. Blood 2010;115:3354-3363.

18. Gregory AD, Capoccia BJ, Woloszynek JR, Link DC. Systemic levels of G-CSF and interleukin-6 determine the angiogenic potential of bone marrow resident monocytes. J Leukoc Biol 2010;88:123-131.

19. Fujikado N, Saijo S, Yonezawa T, Shimamori K, Ishii A, Sugai S, Kotaki H, Sudo K, Nose M, Iwakura Y. Dcir deficiency causes development of autoimmune diseases in mice due to excess expansion of dendritic cells. Nat Med 2008;14:176-180.

20. Hinson ER, Joshi NS, Chen JH, Rahner C, Jung YW, Wang $\mathrm{X}$, Kaech SM, Cresswell P. Viperin is highly induced in neutrophils and macrophages during acute and chronic lymphocytic choriomeningitis virus infection. J Immunol 2010; 184:5723-5731.

21. Miyajima N, Maruyama S, Nonomura K, Hatakeyama S. TRIM36 interacts with the kinetochore protein CENP-H and delays cell cycle progression. Biochem Biophys Res Commun 2009;381:383-387.

22. Nishihashi A, Haraguchi T, Hiraoka Y, Ikemura T, Regnier V, Dodson H, Earnshaw WC, Fukagawa T. CENP-I is essential for centromere function in vertebrate cells. Dev Cell 2002;2:463-476.

23. Vanden Bosch A, Raemaekers T, Denayer S, Torrekens S, Smets N, Moermans K, Dewerchin M, Carmeliet P, Carmeliet G. NuSAP is essential for chromatin-induced spindle formation during early embryogenesis. J Cell Sci 2010;123(Pt 19):3244-3255.

24. Bombardelli L, Carpenter ES, Wu AP, Alston N, DelGiorno KE, Crawford HC. Pancreas-specific ablation of beta1 integrin induces tissue degeneration by disrupting acinar cell polarity. Gastroenterology 2010;138:2531-2540, 2540.e1-4.

25. Ohlsson L, Hjelte L, Huhn M, Scholte BJ, Wilke M, Flod-
strom-Tullberg M, Nilsson A. Expression of intestinal and lung alkaline sphingomyelinase and neutral ceramidase in cystic fibrosis f508del transgenic mice. J Pediatr Gastroenterol Nutr 2008;47:547-554.

26. Kono M, Dreier JL, Ellis JM, Allende ML, Kalkofen DN, Sanders KM, Bielawski J, Bielawska A, Hannun YA, Proia RL. Neutral ceramidase encoded by the Asah2 gene is essential for the intestinal degradation of sphingolipids. J Biol Chem 2006;281:7324-7331.

27. Soundararajan P, Fawcett JP, Rafuse VF. Guidance of postural motoneurons requires MAPK/ERK signaling downstream of fibroblast growth factor receptor 1. J Neurosci 2010;30:6595-6606.

28. Lu X, Su N, Yang J, Huang W, Li C, Zhao L, He Q, Du X, Shen Y, Chen B, et al. Fibroblast growth factor receptor 1 regulates the differentiation and activation of osteoclasts through Erk1/2 pathway. Biochem Biophys Res Commun 2009;390:494-499.

29. Wang SJ, Furusho M, D’Sa C, Kuwada S, Conti L, Morest DK, Bansal R. Inactivation of fibroblast growth factor receptor signaling in myelinating glial cells results in significant loss of adult spiral ganglion neurons accompanied by age-related hearing impairment. J Neurosci Res 2009; 87:3428-3437.

30. Chang R, Wang E. Mouse translation elongation factor eEF1A-2 interacts with Prdx-I to protect cells against apoptotic death induced by oxidative stress. J Cell Biochem 2007;100:267-278.

31. Ruest LB, Marcotte R, Wang E. Peptide elongation factor eEF1A-2/S1 expression in cultured differentiated myotubes and its protective effect against caspase-3-mediated apoptosis. J Biol Chem 2002;277:5418-5425.

32. Roberts SJ, Stewart AJ, Sadler PJ, Farquharson C. Human PHOSPHO1 exhibits high specific phosphoethanolamine and phosphocholine phosphatase activities. Biochem J 2004;382(Pt 1):59-65.

33. Roberts SJ, Stewart AJ, Schmid R, Blindauer CA, Bond SR, Sadler PJ, Farquharson C. Probing the substrate specificities of human PHOSPHO1 and PHOSPHO2. Biochim Biophys Acta 2005;1752:73-82.

34. Yoshida H, Russell J, Granger DN. Thrombin mediates the extraintestinal thrombosis associated with experimental colitis. Am J Physiol Gastrointest Liver Physiol 2008; 295:G904-G908. 